\title{
MATRIX INFLUENCE ON DETERMINATION OF ORGANOCHLORINE PESTICIDE RESIDUES IN WATER BY SOLID PHASE EXTRACTION COUPLED TO GAS CHROMATOGRAPHY-MASS SPECTROMETRY
}

\author{
C. RIMAYI ${ }^{1}$, D. ODUSANYA ${ }^{1}$, F. MTUNZI ${ }^{2} \&$ C. VAN WYK ${ }^{3}$ \\ ${ }^{1}$ Department of Water Affairs, Resource Quality Services (RQS), Roodeplaat, South Africa. \\ ${ }^{2}$ Department of Chemistry, Vaal University of Technology, Vanderbijlpark, South Africa. \\ ${ }^{3}$ Department of Biotechnology, Vaal University of Technology, Vanderbijlpark, South Africa.
}

\begin{abstract}
The presence of a sample matrix is one of the most important practical considerations in gas chromatography analysis as there are potentially numerous problems associated with matrix based injections. This paper aims to highlight the distinction between blank sample analysis and real sample analysis using automated solid phase extraction (SPE) and gas chromatography-mass spectrometry. Four reversed sorbent phases, including a Supelco LC-18, Strata C-18-E and Strata-X (styrene divinyl benzene) were used for SPE method development using an automated Gilson GX-271 Aspec ${ }^{\mathrm{TM}}$ liquid handling instrument to determine the best solid phase and treatment for optimum organochlorine determination. The method developed proved to be valid when tested against parameters such as calibration range, coefficient of regression, linearity, repeatability and sensitivity. The StrataX and LC-18 cartridges produced the best recoveries, varying between $90 \%$ and $130 \%$ for most analytes. The LC- 18 was selected for further analysis of the matrix effects as it showed greater reproducibility and method parameter robustness. Various real matrix sample volumes were tested on the selected LC-18 cartridge to determine its optimum maximum matrix load for efficient recoveries (breakthrough volume equivalent). A $100 \mathrm{ml}$ sample volume was determined as the optimum matrix load volume as it produced more precise recoveries than other spiked sample matrix volumes. Visual comparison and analysis of selective ion monitoring chromatograms of both matrix based and matrix-free extracts indicate that there are significant matrix effects potentially capable of adversely affecting the chromatographic system from producing accurate identification and quantification of target analytes.
\end{abstract}

Keywords: Gas chromatography-mass spectrometry, matrix effects, solid phase extraction.

\section{INTRODUCTION}

The development of a selective solid phase extraction (SPE) analytical method depends on the ability of the sorbent phase to selectively isolate the analytes of interest and eliminate the sample matrix from the final extract [1]. The presence of a sample matrix presents a host of challenges pertaining to the quantification and detection of analytes [2, 3]. Sample cleanup and concentration are essential for reaching low detection limits [4]. The development of multi-class pesticide residue analytical method, coupled to automation of the SPE step goes a long way to increase sample throughput. The analysis of organochlorine compounds involves isolation of the analytes from the sample matrix, removal of matrix components, followed by the identification and quantification of the target analytes [5]. Multi-residue analysis has been identified as a cost effective and labour saving method for determination of a wide range of analytes within a single run, but obtaining optimum recoveries for all analytes is practically impossible $[6,7]$. The wider the physico-chemical properties the analytes within a cocktail mixture have, the more difficult it is to obtain overall optimum recoveries for all the analytes within a single run [5]. 
The aim of any gas chromatography-mass spectrometry (GC-MS) analytical procedure is to achieve resolution and positive identification of the analytes of interest. Common GC-MS is prone to detect co-extracted matrix components and hence must be configured for optimum selectivity as even the selective ion monitoring (SIM) and tandem (MS/MS) modes are also prone to matrix interference [8]. The use of inert material such as deactivated glass liners and solid phases, appropriate injection techniques, carbon frits in the injection liner and the addition of analyte protectants to the final extract may lower the matrix effects but do not eliminate them [8].

The principle of SPE involves the partitioning of the analytes between 2 phases, that is the water sample and the solid phase [9]. The SPE method development aimed to establish a method that it specific and selective to the organochlorine pesticides. The SPE sample preparation step is most important in eliminating the matrix components responsible for the matrix effects and is the principal source of imprecision and inaccuracy [9]. The process may however be time-consuming and may lead to substantial loss of analytes, leading to lower recoveries and higher uncertainties being reported with the analytical results [8].

\subsection{Matrix effects in GC-MS}

The matrix effects in GC-MS can be defined as the effect of co-eluting residual matrix components on the resolution, selectivity and ionisation of the target analytes [10]. They result in either signal suppression or enhancement [5]. Some of the factors which influence matrix effects include the nature and amount of both the matrix components and analytes, the type of detector used, the surface activity and geometry of the injection liner, column dimensions and the efficiency of maintenance of the entire GC-MS chromatographic system $[11,12]$. The most obvious way to reduce the matrix effects is to reduce the amount of matrix components entering the chromatographic system. The matrix cannot be entirely eliminated from the extracts and are unavoidably present in analysed samples $[6,13,14]$. Methods of taking the matrix effect into account have been studied but they do not necessarily reduce its influence $[15,16]$. Accounting for the matrix effects in principle can lead to corrected results, but for methods which undergo stronger ionisation suppression, its efficiency is limited [17]. Furthermore, since the nature and amount of these co-eluting compounds are usually variable between samples, the matrix effects can be highly variable and difficult to predict, making it difficult to compensate for them in practice $[18,19]$. Whilst different techniques can be applied to compensate for the matrix effects and produce quantitatively accurate results, the loss in method sensitivity that is accompanied by signal suppression and the variability in method sensitivity that occurs between samples cannot be eliminated [6, 20, 21].

When matrix based injections are introduced into the GC-MS, the matrix components are mainly retained in the injection liner and first metre of the capillary column. The matrix components retained in the active sites lead to an increase in the amount of analytes reaching the detector, as the matrix components compete with the analytes for the active sites and occupy the active sites which would otherwise be occupied by the analytes [22]. This leads to an increased response and subsequently matrix induced enhanced responses [8]. Matrix components also protect the target analytes from decomposition in the hot injector by lowering the eutectic temperature of the target analytes of interest, leading to matrix induced enhanced 
chromatographic effects. This phenomenon is used to explain recovery values which exceed $100 \%$ [6].

Gradual deposition and accumulation of non-volatile matrix components in the chromatographic system leads to an increase in the number of active sites. The presence of active sites in the chromatographic system, especially the injection liner presents problems associated with matrix-induced diminished response chromatographic effects due to the resulting adsorption or decomposition of analytes [5]. The matrix component peak may also partially or completely mask the analyte peak of interest at a specific retention time, leading to inaccurate quantification. The matrix effect strongly depends on the nature of the analyte and on the properties of the co-eluting compounds, as some of the co-eluting compounds elute as chromatographic peaks and cause ionisation efficiency change only in a limited retention time range [6]. The resulting matrix effects may also cause inaccurate false positive and negative results. The use of matrix matched standards may be employed to correct some of the matrix effects to produce more precise analytical determinations [23].

\section{EXPERIMENTAL}

The automated analytical methodology developed and presented in this paper was aimed for further research towards studying the matrix effects.

\subsection{Materials and methods}

Grade A volumetric flasks and pipettes, funnels, spatula, Pasteur pipettes, vials and inserts were used for reagent preparation. Methanol, dichloromethane (DCM), toluene, acetone, hexane, SPE cartridges, collection vials, $2 \mathrm{ml}$ vials and caps, test tubes, nitrogen gas were also used in the SPE method development. A Mettler Loledo AX105 Delta Range ${ }^{\circledR}$ analytical balance was used to weigh the standards to 3 decimal places.

\subsection{Quality control}

All volumetric flasks and pipettes were calibrated before use. Analytical balances were calibrated annually and verified using reference masses daily. Grade A volumetric glassware and analytical (pesticide) grade reagents were also used for the entire analysis with a purity $>99 \%$. All cartridge testing for SPE method development was done in at least duplicate analysis. Deionised ultrapure water was sourced from a Millipore Milli-Q system. The water was passed through an organic compound scavenger resin bed before passing to the Millipore Milli-Q system. The certified pesticide neat standards had a purity of at least $98.5 \%$ (obtained from Dr Ehrenstorfer and Chemservice) and $100 \mathrm{mg} / \mathrm{l}$ stock solution and subsequent cocktails were prepared in toluene and stored at $\leq-18^{\circ} \mathrm{C}$. Spiking solutions were prepared in acetone. Temperatures for the laboratory atmosphere and freezers were monitored daily.

\section{GC-MS CONFIGURATION}

An Agilent Technologies 6890 GC coupled to an Agilent Technologies 5975 quadrupole mass selective detector was used for the analysis, using a $30 \mathrm{~m} \times 0.25 \mathrm{~mm} \times 0.25 \mu \mathrm{m}$ DB-5MS column with stationary phase $5 \%$ phenyl and $95 \%$ dimethylpolysiloxane. The mobile phase of choice used was $99.999 \%$ helium gas supplied by Airliquide South Africa. 
Total runtime for the analysis was 31.87 minutes with initial temperature of $70^{\circ} \mathrm{C}$ and hold time of 2 minutes. Ramp 1 was $25^{\circ} \mathrm{C} / \mathrm{min}$ to $150^{\circ} \mathrm{C}$, with no hold time. Ramp 2 was $3^{\circ} \mathrm{C} / \mathrm{min}$ to $200^{\circ} \mathrm{C}$, with no hold time and ramp 3 was $8^{\circ} \mathrm{C} / \mathrm{min}$ to $280^{\circ} \mathrm{C}$ with no hold time. A constant pressure of $129.9 \mathrm{KPa}$ was maintained with an average velocity of $50 \mathrm{~cm} / \mathrm{second}$. Data was analysed using Chemstation software from Agilent Technologies. A $1 \mu$ volume of sample was injected using a Gerstel MP2 twister autosampler.

\section{PEAK IDENTIFICATION}

SIM mode was configured into the GCMS for greater selectivity and sensitivity. An average of 4 major ion fragments from each analyte was selected for use in identification of the compounds which are displayed in Table 1.

All the peaks from the $1 \mathrm{ppm}$ cocktail mix having been identified, calibration standards were then made up by serial dilution for validating the GCMS instrument method, using the calibration levels: $1 \mathrm{ppm}, 0.5 \mathrm{ppm}, 0.25 \mathrm{ppm}, 0.125 \mathrm{ppm}, 0.0625 \mathrm{ppm}, 0.0313 \mathrm{ppm}$, $0.0156 \mathrm{ppm}$ and $0.0078 \mathrm{ppm}$. The $1 \mathrm{ppm}$ cocktail was also used to test SPE cartridges for efficiency of extraction and determination of validation criteria for the SPE method.

Table 1: Target and qualifier ions used for SIM analysis.

\begin{tabular}{clccccc}
\hline Peak No. & Peak name & $(\mathrm{min})$ & $\mathrm{T}$ & $\mathrm{Q} 1$ & $\mathrm{Q} 2$ & $\mathrm{Q} 3$ \\
\hline 1 & Pentachlorobenzene & 8.96 & 250 & 108 & 213 & 252 \\
2 & BHC-alpha & 12.08 & 181 & 183 & 217 & 219 \\
3 & Hexachlorobenzene & 12.431 & 284 & 249 & 142 & 214 \\
4 & BHC-beta & 13.194 & 181 & 109 & 219 & 217 \\
5 & Lindane (BHC-gamma) & 13.444 & 181 & 217 & 109 & 219 \\
6 & PCNB & 13.705 & 237 & 295 & 249 & 239 \\
7 & BHC-delta & 14.505 & 183 & 219 & 217 & 109 \\
8 & Heptachlor & 16.824 & 272 & 237 & 337 & 135 \\
9 & Aldrin & 18.578 & 263 & 293 & 66 & 186 \\
10 & Heptachlor-epoxide & 20.765 & 353 & 237 & 263 & 253 \\
11 & trans-Chlordane (gamma) & 22.076 & 375 & 272 & 237 & 263 \\
12 & Endosulfan alpha & 22.663 & 170 & 241 & 195 & 265 \\
13 & cis-Chlordane (alpha) & 22.868 & 373 & 272 & 263 & 339 \\
14 & Dieldrin & 23.899 & 263 & 277 & 265 & 108 \\
15 & 4,4'-DDE & 24.059 & 246 & 318 & 176 & 316 \\
16 & Endrin & 24.753 & 263 & 245 & 81 & 317 \\
17 & Endosulfan beta & 25.178 & 195 & 237 & 265 & 159 \\
18 & 4,4'-DDD & 25.738 & 235 & 237 & 165 & 199 \\
19 & Endosulfan SO & 26.783 & 272 & 229 & 387 & 237 \\
20 & 4,4'-DDT & 27.009 & 235 & 237 & 199 & 165 \\
21 & Mirex & 29.887 & 272 & 274 & 237 & 332 \\
\hline
\end{tabular}

T, Target ion; Q, Qualifier ion; 4,4' DDD, 4,4' Dichlorodiphenyldichloroethane; 4,4' DDE, 4,4' Dichlorodiphenyldichloroethylene; 4,4' DDT, 4,4' Dichlorodiphenyltrichloroethane; PCNB, Pentachloronitrobenzene. 


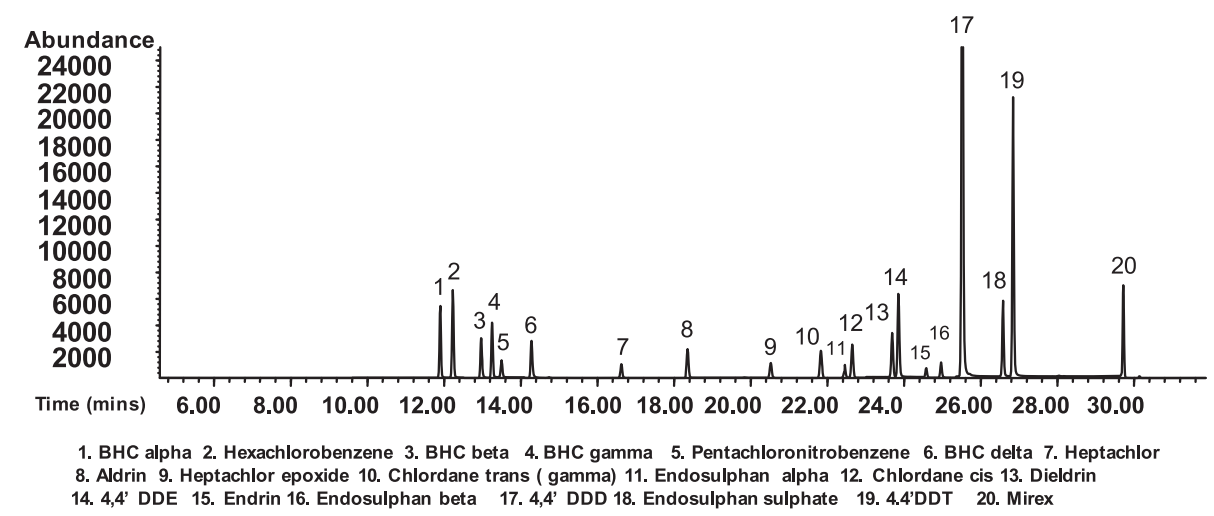

Figure 1: SIM chromatogram of organochlorine cocktail.

\section{SPE TEST PROCEDURE}

The automated SPE method development was designed to configure the best procedure to use for the extraction of the organochlorine compounds under study. The test procedure considered the following parameters;

1. Sorbent (solid phase) choice

2. Sorbent treatment

3. Sorbent mass

4. Sample pre-treatment

5. Sample volume and flow mechanism

6. Solvent choice

The Gilson GX-271 Aspec ${ }^{\mathrm{TM}}$ liquid handling instrument was used to condition cartridges, load samples onto the cartridges and also to dry and elute the cartridges. Automation the SPE process is advantageous in that it leads to higher sample throughput, saves time, and improves accuracy and precision whilst substantially reducing the chances of human error [24, 25].

A consistent flow rate at low pressure was applied as it is recommended for effective mass transfer of analytes onto the sorbent phase $[25,26]$. The Gilson GX-271 Aspec ${ }^{\mathrm{TM}}$ liquid handling instrument utilises positive pressure elution which makes it increasingly easy to control flow rates [27]. Extensive cleanup of extracts may result in the partial loss of some compounds, hence this method development was aimed at retaining as much analyte as possible within the final extract [6].

\section{RESULTS AND DISCUSSION}

\subsection{GCMS instrument method validation}

Method validation is essential as it confirms that an analytical method is effective in measuring the parameters it is intended to measure. Successful validation of this instrument method validation will confirm that the methods, procedures and protocols applied in the analysis produce reliable and accurate data and also ensure that valid conclusions are postulated as a result of the validated method [28]. 


\subsection{Validation parameters}

For the purposes of method validation the parameters tested were linearity, linearity verification by excel, working range, repeatability, reproducibility, limits of detection (LOD), limits of quantification (LOQ) and analysis of variance.

\subsubsection{Linearity}

Eleven independent calibration curves were prepared for the purposes of validating the linearity of each analyte. The results are displayed in Table 2.

For most instruments, a linear response is expected from calibration standards made by serial dilution from a stock solution. Not all compounds analysed by GC-MS display a linear fit on calibration though, this according to Soboleva et al. [29], can largely be attributed to either re-isomerisation, decomposition or transformation of the target analytes, either within the chromatographic system or before introduction into the chromatographic system. This leads to greater uncertainties in GC measurements. The MS detector responds to changes in the sample concentration then displays a nonlinear fit. Previous studies have shown that, this loss of linearity in most compounds may largely be attributed to breakdown of the compounds due to high GC oven temperature [30]. Other researchers indicate that the breakdown of the compounds increases as the GC oven temperature increases hence it is better to start off with lower GC oven temperatures on analysis. Choi et al. [31] stated that contaminants from sample processing or analyte extraction from physiological matrices can be ionised together with the compound of interest, causing a phenomenon called matrix signal suppression effects. This effect can lead to loss of linearity especially if the samples are sandwiched evenly in between the standards during a sequence run.

For the purposes of this study, a linear curve graph displaying a regression of $\geq 0.998$ with at least four calibration levels was considered to be significantly linear. A total of seven calibration levels were used to test the linearity of the fit and also to determine the calibration range. The linear curve does not always pass through the origin as this characteristic depends on the detection limit. The calibration curves show that the lower the detection limit, the closer the curve is to the graph origin $(0,0)$.

The regression data displayed in bold shows calibration curves with a nonlinear fit and those in nonbold show a linear regression. It can be deduced from the above data that aldrin, hexachlorobenzene-alpha (BHC-alpha), BHC-beta, BHC-delta, BHC-gamma, cis-chlordane (beta), trans-chlordane (gamma), dichlorodiphenyldichloroethylene (4,4'-DDE), dieldrin, endosulphan alpha, endosulphan beta, BHC and mirex all display distinct linear fit. Although endrin, Dichlorodiphenyldichloroethane (4,4'-DDD), endosulphan $\mathrm{SO}_{4}$, heptachlor epoxide, and heptachlor displayed between two and one nonlinear calibration curves, it can also be overwhelmingly deduced that they also show a linear fit.

Experience shows that problems associated with obtaining a nonlinear calibration fit lie mainly within the chromatographic system [29]. Previous studies by Scientific Services Inc. have proved that the selection of the liner type, liner packing type, liner packing position, solvent volume used, injection volume used, injection technique, and oven temperatures used all have a profound effect on the linearity of a calibration fit of a specific compound.

The validity of the Chemstation software for computing the regression was determined by calculating the regression using Microsoft Excel. The results in Table 3 below indicate that the Chemstation software was indeed effective in regression calculation. Minor differences in the resultant regression values between Chemstation and Excel were expected as Chemstation 


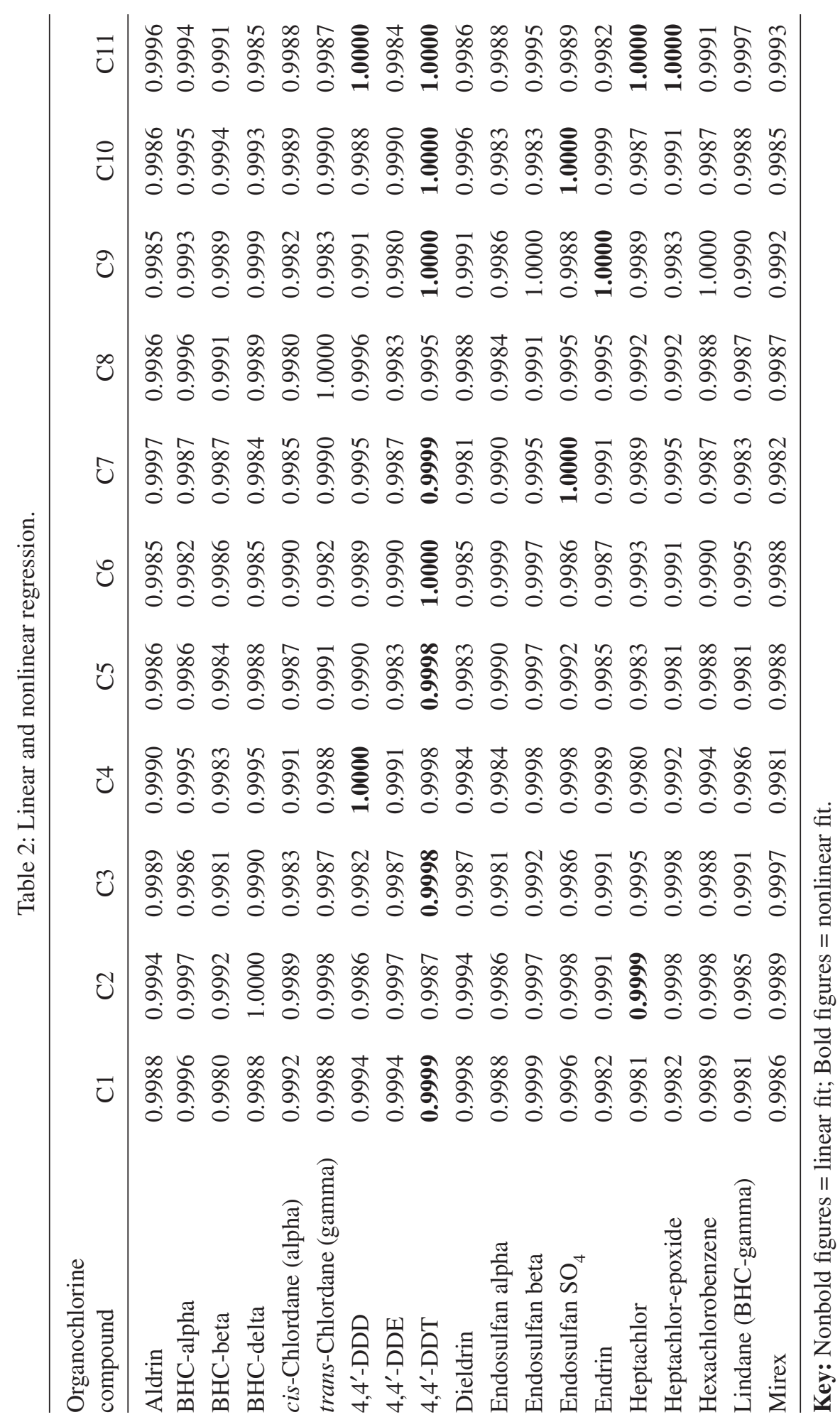




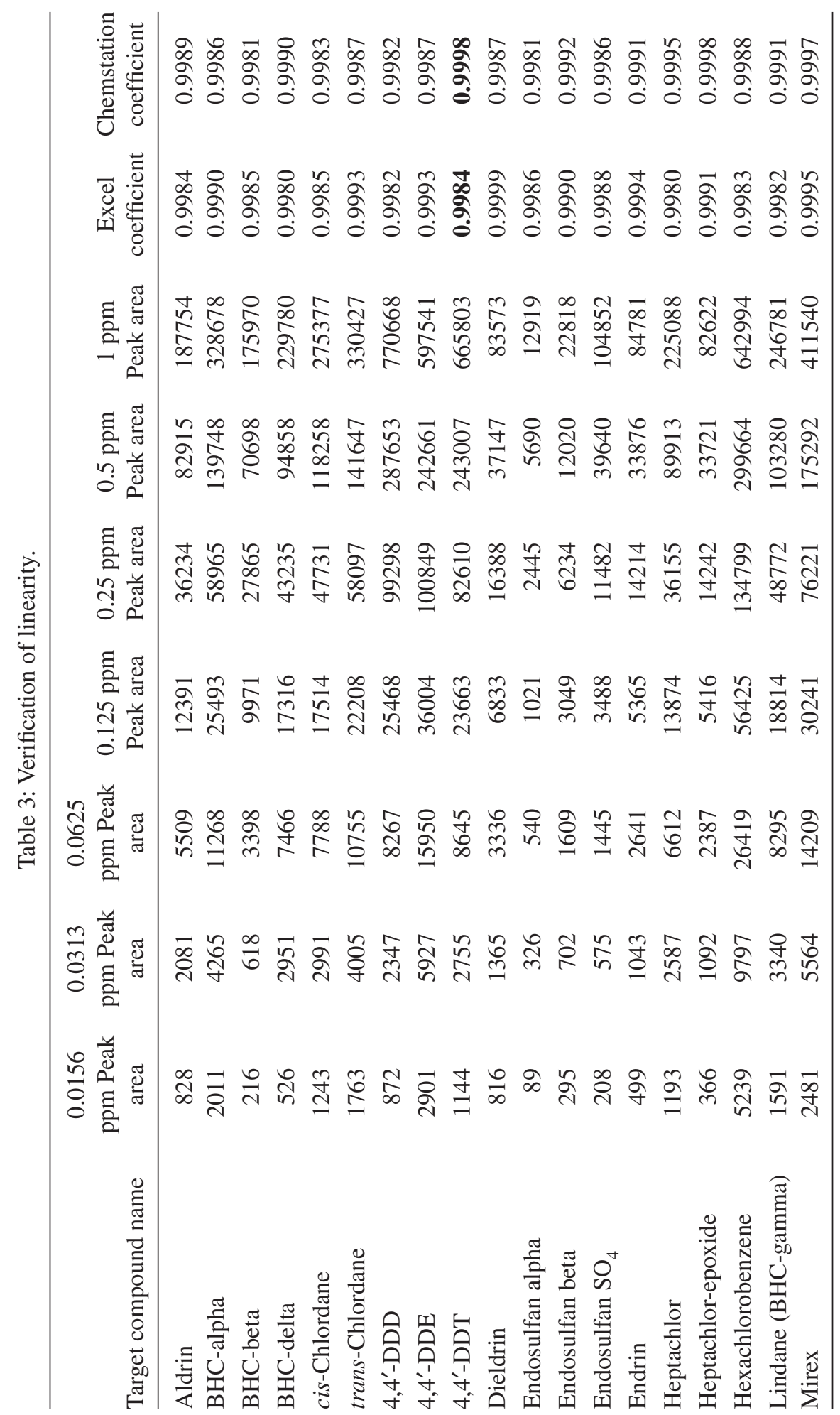


Table 4: Calibration ranges for selected organochlorine compounds.

\begin{tabular}{llcccc}
\hline Compound name & $\begin{array}{c}\text { Linear } \\
\text { range }\end{array}$ & \multicolumn{1}{c}{$\mathrm{R}^{2}$} & $\begin{array}{c}\text { Repeatability } \\
(\mathrm{RSD} \%)\end{array}$ & LOD & LOQ \\
\hline Aldrin & $1-0.0313$ & 0.9990 & 6.45 & 0.038 & 0.125 \\
BHC-alpha & $1-0.125$ & 0.9984 & 9.63 & 0.062 & 0.205 \\
BHC-beta & $1-0.0313$ & 0.9985 & 3.92 & 0.060 & 0.201 \\
BHC-delta & $0.5-0.0313$ & 0.9980 & 4.59 & 0.018 & 0.060 \\
cis-Chlordane (alpha) & $1-0.0313$ & 0.9995 & 3.22 & 0.048 & 0.161 \\
trans-Chlordane (gamma) & $1-0.125$ & 0.9993 & 3.23 & 0.049 & 0.162 \\
4,4'-DDD & $1-0.125$ & 0.9982 & 5.93 & 0.083 & 0.277 \\
4,4'-DDE & $1-0.0156$ & 0.9993 & 4.31 & 0.068 & 0.228 \\
4,4'-DDT & Nonlinear & Nonlinear & 5.25 & 0.078 & 0.261 \\
Dieldrin & $0.5-0.0313$ & 0.9999 & 3.75 & 0.025 & 0.082 \\
Endosulfan alpha & $1-0.0313$ & 0.9986 & 4.23 & 0.047 & 0.155 \\
Endosulfan beta & $1-0.0156$ & 0.9990 & 2.65 & 0.019 & 0.063 \\
Endosulfan SO & $1-0.25$ & 0.9988 & 7.17 & 0.027 & 0.091 \\
Endrin & $0.5-0.125$ & 0.9994 & 5.81 & 0.021 & 0.071 \\
Heptachlor & $0.5-0.125$ & 0.9980 & 5.17 & 0.037 & 0.123 \\
Heptachlor-epoxide & $0.5-0.0156$ & 0.9991 & 4.39 & 0.029 & 0.095 \\
Hexachlorobenzene & $0.5-0.0625$ & 0.9983 & 2.75 & 0.023 & 0.077 \\
Lindane (BHC-gamma) & $0.5-0.0313$ & 0.9982 & 4.20 & 0.018 & 0.059 \\
Mirex & $1-0.125$ & 0.9995 & 4.41 & 0.056 & 0.187 \\
\hline
\end{tabular}

overtly has advantages in manipulating plots such as forcing the curve through the origin so as to improve the quantification of analytes just near the detection limits, an action which is usually detrimental to achieving better coefficients of regression. It should however be noted that the coefficient displayed for dichlorodiphenyltrichloroethane $\left(4,4^{\prime}-\mathrm{DDT}\right)$ is the regression for the quadratic fit.

The above Table 4 shows the calibration range in which acceptable accuracy, linearity and precision can be obtained.

\subsubsection{Calibration range}

The calibration range for the selected organochlorine compounds were tested using $1 \mathrm{ppm}$, $0.5 \mathrm{ppm}, 0.25 \mathrm{ppm}, 0.125 \mathrm{ppm}, 0.0625 \mathrm{ppm}, 0.0313 \mathrm{ppm}, 0.0156 \mathrm{ppm}$ and $0.0078 \mathrm{ppm}$. The $0.0078 \mathrm{ppm}$ standard was then rejected as it was extremely difficult to distinguish between background (noise) peaks and the analyte peaks for most compounds. Table 4 shows that $4,4^{\prime}$-DDE, and endosulfan beta had the broadest linear ranges of $1 \mathrm{mg} / \mathrm{l}$ to $0.0156 \mathrm{mg} / \mathrm{l}$.

\subsubsection{Precision}

Precision is the measure of the degree of repeatability on an analytical method under normal operation. For ease of reference, precision was categorised into repeatability and reproducibility [32]. 


\subsubsection{Repeatability}

An exact value of precision is not easy to estimate practically as a correct estimate cannot be obtained until the same experiments are repeated many times [33]. Repeatability was computed as a function of percentage relative standard deviation (\%RSD). A 1 ppm standard was analysed 11 times to determine the percentage relative standard deviation.

$$
\% \mathrm{RSD}=\frac{\text { Standard deviation }}{\text { Mean }} \times 100
$$

As a quality control procedure, \%RSD of less than $10 \%$ is considered to be valid. Accordingly all analytes tested showed a percentage RSD of less than $10 \%$.

\subsubsection{Sensitivity}

The GC-MS sensitivity was validated against LOD and LOQ. LOD is the lowest detectable concentration with a signal to noise ratio of at least 3 whilst LOQ is the lowest quantifiable concentration with a signal to noise ratio of at least 10 [7, 34, 35]. Both the LOD and LOQ were computed statistically with as three and ten times the standard error of the calibration curve respectively. Most analytes displayed a significant degree of sensitivity with BHC-delta showing the lowest LOD and LOQ of $0.018 \mathrm{mg} / \mathrm{l}$ and $0.059 \mathrm{mg} / \mathrm{l}$ respectively.

\section{SPE METHOD VALIDATION}

Sample preparation removes a major part of the matrix components, but a small amount often remains in the treated sample possibly inducing matrix effects [19]. Validation of the sample extraction SPE method is therefore essential to determine the presence and impact of the matrix components in quantification of the target analytes.

The first parameter tested on the three cartridges, namely Strata-C-18-E $200 \mathrm{mg}$, (Supelco) LC-18 $200 \mathrm{mg}$ and Strata-X $500 \mathrm{mg}$ was the effect of conditioning versus not conditioning of the cartridges. The Table 5 shows the responses and concentration obtained when $2 \mathrm{ml}$ of a $1 \mathrm{ppm}$ solution was loaded onto the unconditioned cartridges and the elute obtained after loading was analysed on the GC-MS. The results indicate that no analytes were retained at all by the cartridges when the cartridges were not conditioned.

The data in Table 5 indicates that the elute showed a higher response and concentration than the original 1 ppm concentration initially loaded onto the cartridge. This shows the phenomenon called matrix induced enhanced chromatographic effects and it also explains the rationale for poor accuracy for some data generated by routine GC methods employing traditional calibration strategies for quantification of analytes [5, 12]. External calibration methods of injecting a cocktail of neat calibration standards were performed for quantification and the results indicate that the synthetic matrix indeed had an effect on the quantification of the analytes after elution even in the absence of a real sample matrix.

\subsection{Results of conditioned cartridges}

$2 \mathrm{ml}$ of a $1 \mathrm{ppm}$ cocktail solution of the organochlorine compounds was loaded onto a cartridge previously conditioned using $2 \mathrm{ml}$ methanol. The elute collected after loading was analysed by GC-MS. The results of GC-MS analysis of the elute displayed in Fig. 2 indicate that there was significant analyte retention by all cartridges, particularly the LC-18 and Strata $\mathrm{X}$ cartridges whose results indicate that significant quantities of the analytes were 


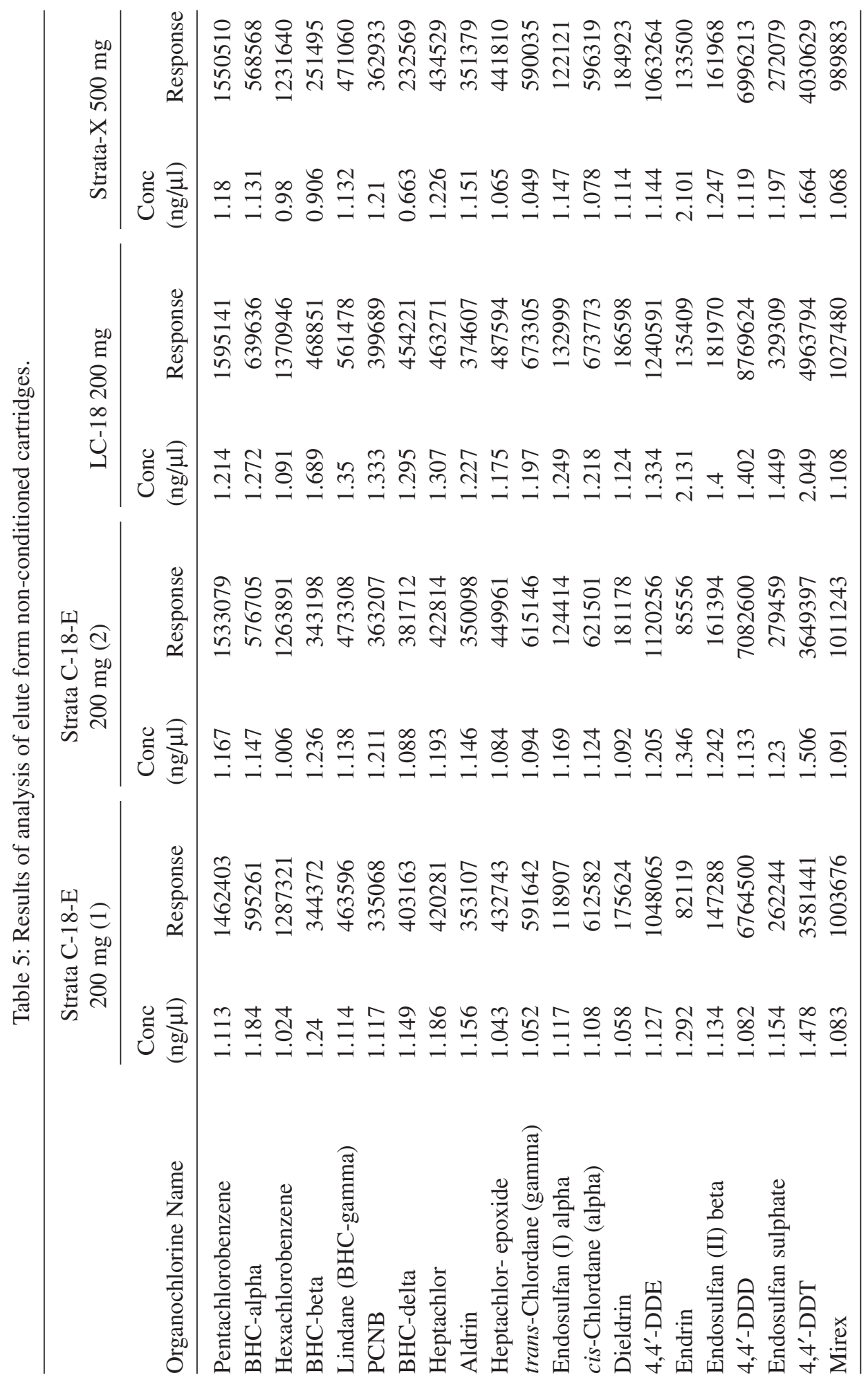




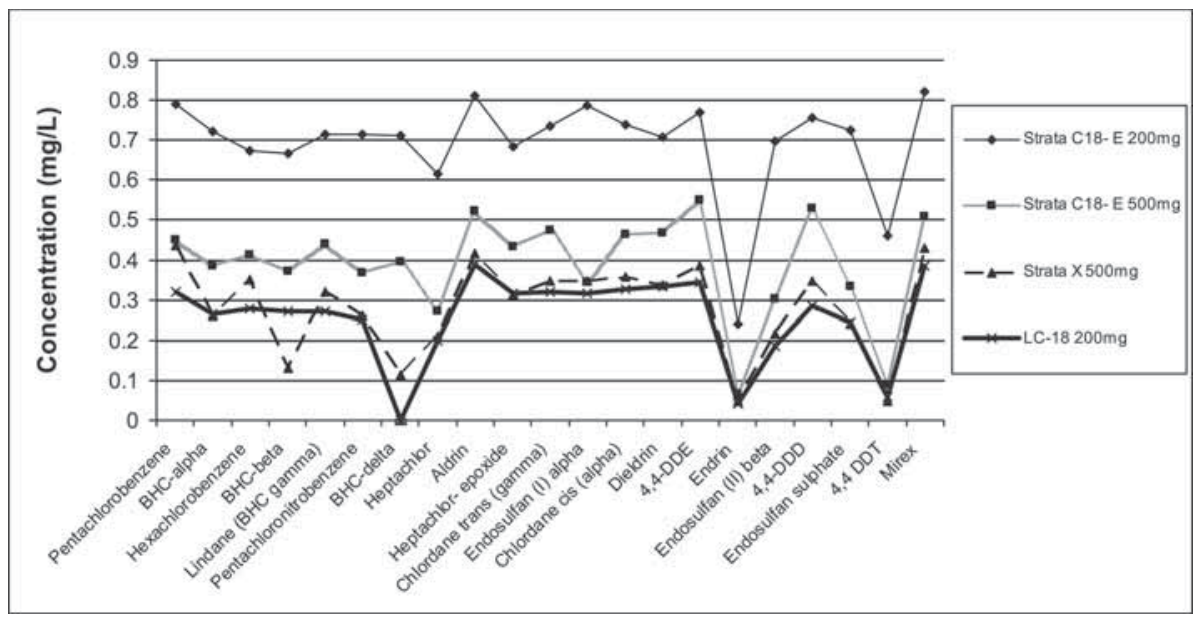

Figure 2: Results of conditioned cartridges.

adsorbed by the solid phase as shown by low analyte concentrations detected within the elute. Most notably is the absence of BHC-delta in the elute extracted using the LC-18 cartridge indicating that there was up to $100 \%$ retention. It can therefore be deduced that that is essential to condition the cartridges before use, with the degree of conditioning depending upon the nature of the sorbent bed and the bed mass. These results are in sync with findings by Poole et al. [25] who postulated that the high surface tension of water often causes slow and uneven flow rates through solid phases when cartridges are not conditioned first before loading the sample, resulting in low analyte recovery.

By comparing the cartridges Strata C-18-E $200 \mathrm{mg}$ with the Strata C-18-E $500 \mathrm{mg}$ in Fig. 2 above, it can be deduced that to some extent increasing the sorbent bed mass leads to an increase the degree of analyte retention significantly. From the results in Fig. 3, it can be deduced that the LC-18 cartridge is more efficient in retaining the organochlorine compounds as the elute overall showed the lowest analyte concentration within the elute.

\subsection{Recovery of test cartridges}

Most researchers decline to indicate their acceptable recoveries especially for matrix based determinations as it is difficult to maintain strict recovery targets particularly when the nature of the matrix under study is unknown. Poole [36], however, indicated that recoveries above $90 \%$ were acceptable. For this research, recoveries of $100 \pm 30 \%$ for determinations were considered to be acceptable. Figure 3 below indicates that the LC-18 and Strata X cartridges showed the best recoveries, although some of the method development parameters are yet to be optimised in order to meet the target of $100 \pm 30 \%$.

\subsection{Effect of conditioning volume on analyte retention}

Figure 4 above shows the results obtained after conditioning the cartridges with both $2 \mathrm{ml}$ and $6 \mathrm{ml}$ methanol, followed by loading $2 \mathrm{ml}$ of $0.4 \mathrm{ppm}$ cocktail solution before collecting the 


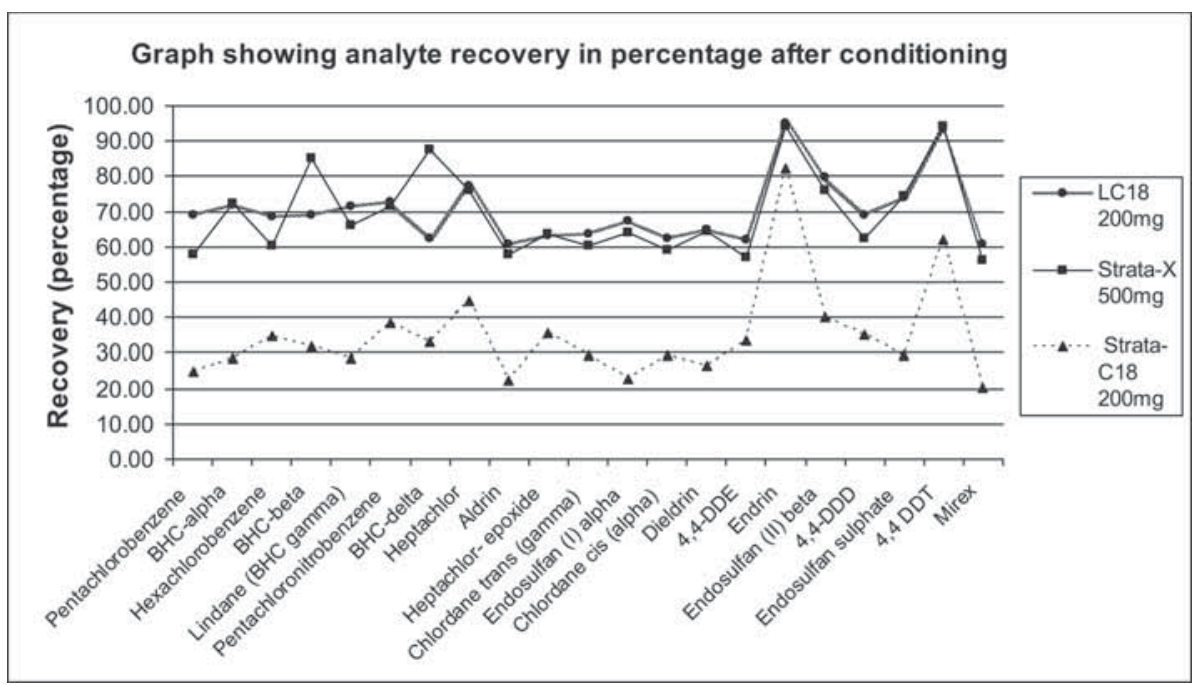

Figure 3: Results of analyte recovery after conditioning in percentage.

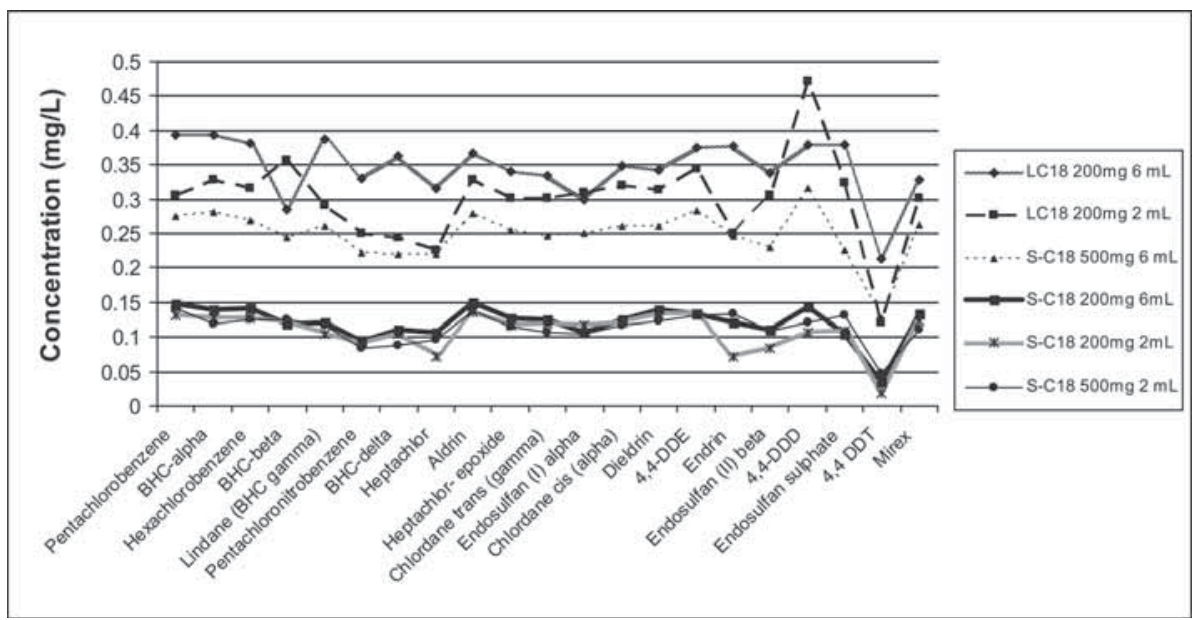

Figure 4: Effect of conditioning volume on analyte retention.

elute to a $2 \mathrm{ml}$ final volume. The elute collected was analysed by GC-MS and the results show that the volume of conditioning solvent used has a significant effect on the efficiency of analyte retention of the cartridges. The conditioning volume had a greater effect on the SC-18 E $500 \mathrm{mg}$ cartridges as it shows a greater difference in analyte retention when the conditioning volume is increased from $2 \mathrm{ml}$ to $6 \mathrm{ml}$. Once again the LC-18 displays the greatest efficiency and robustness as the increase from $2 \mathrm{ml}$ to $6 \mathrm{ml}$ does not have as much significant effect on the efficiency of analyte retention compared to other cartridges. Conditioning of the LC-18 cartridge with $6 \mathrm{ml}$ methanol proved to provide the optimum cartridge performance and considerably increased the recovery of most analytes to the target of $100 \pm 30 \%$. 


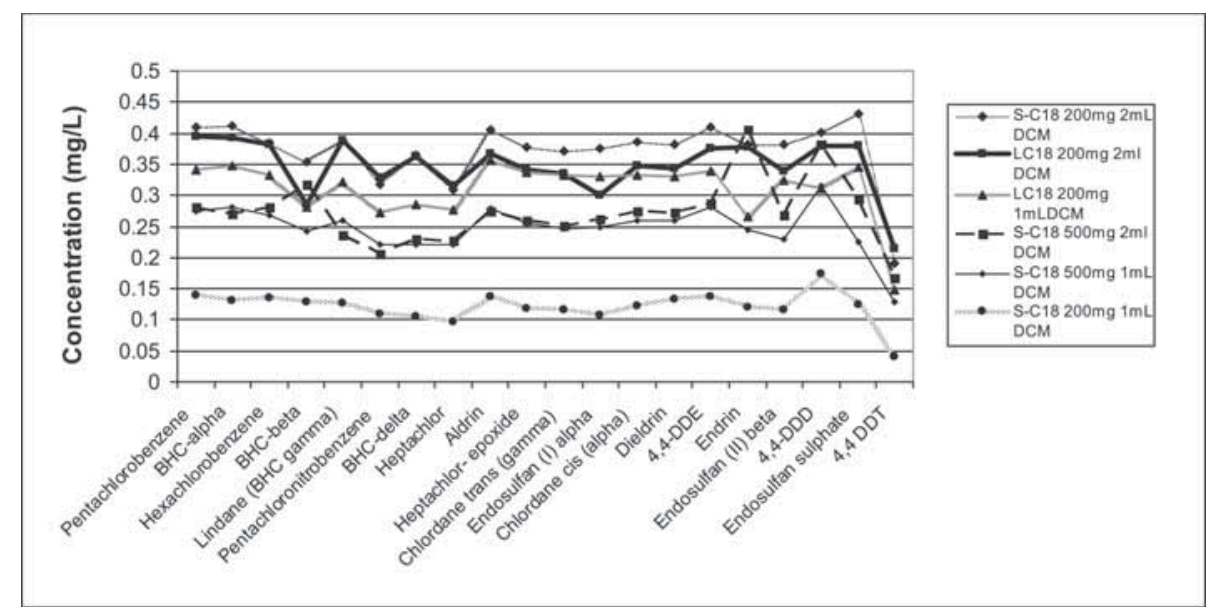

Figure 5: Effect of elution volume on analyte retention.

\subsection{Effect of elution volume on cartridge efficiency}

Figure 5 above shows the effect of the elution volume tested on the LC-18 $200 \mathrm{mg}$, SC-18 E $200 \mathrm{mg}$ and SC-18 E $500 \mathrm{mg}$ cartridges. The cartridges were firstly conditioned with $6 \mathrm{ml}$ methanol before loading with $1 \mathrm{ml}$ of a $0.4 \mathrm{ppm}$ organochlorine cocktail. Increasing the elution volume twofold from $1 \mathrm{ml}$ to $2 \mathrm{ml}$ produced considerable changes in the amount of analytes desorbed from the sorbent bed, particularly for the SC-18 E $200 \mathrm{mg}$ and LC-18 $200 \mathrm{mg}$ cartridges. This indicates that $1 \mathrm{ml}$ eluent was insufficient to desorb all analytes from the sorbent bed. $2 \mathrm{ml} \mathrm{DCM}$ was found to be optimally capable of desorbing most analytes from the LC-18 $200 \mathrm{mg}$ and SC-18 E $200 \mathrm{mg}$ cartridges. Subsequent analysis of further $2 \mathrm{ml}$ aliquots on the same cartridges proved that $6 \mathrm{ml}$ DCM was the most efficient volume required to desorb any remaining analyte traces from the solid phase. The above data also shows that in some cases, increasing the sorbent mass does not necessarily lead to greater cartridge efficiency as the SC-18 E $200 \mathrm{mg}$ cartridge proved to be more efficient than the SC-18 E $500 \mathrm{mg}$ cartridge. Furthermore, increasing the elution volume for the SC-18 E $500 \mathrm{mg}$ cartridge from $1 \mathrm{ml}$ to $2 \mathrm{ml}$ produced no significant difference.

\subsection{Optimised sample preparation technique}

The matrix is a burden on pesticide residue analysis [22]. Unfortunately it is presently impossible to completely eliminate the matrix from a real sample matrix in order to isolate the analyte of interest [37]. Dedicated SPE application techniques have been developed to give with extracts with comparatively low matrix burden but several problems still arise in the GC analysis of the pesticide residues [22,38]. The following sample preparation conditions were developed for optimum analyte extraction and recovery:

1. Condition with $6 \mathrm{ml}$ methanol with flow rate $6 \mathrm{ml} / \mathrm{min}$

2. Load $10 \mathrm{ml}$ sample with flow rate $1.5 \mathrm{ml} / \mathrm{min}$

3. Dry using nitrogen gas for 2 minutes with flow rate $6 \mathrm{ml} / \mathrm{min}$

4. Elute with $6 \mathrm{ml} \mathrm{DCM}$ with rate $1.5 \mathrm{ml} / \mathrm{min}$. 


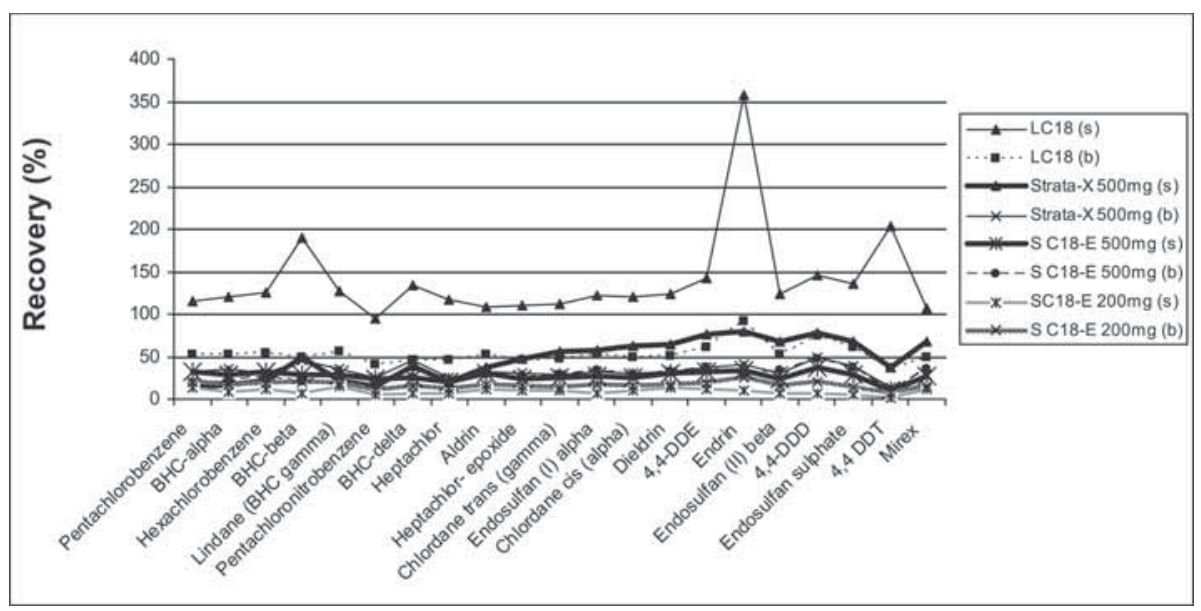

Figure 6: Recovery of spiked sample and blank water.

\subsection{Results of real sample and blank analysis}

Each of the four test cartridges were individually loaded with both $10 \mathrm{ml}$ real sample water (s) spiked with a $1 \mathrm{ppm}$ organochlorine cocktail solution and another four with $10 \mathrm{ml}$ blank deionised water (b) spiked with a $1 \mathrm{ppm}$ organochlorine cocktail solution. The samples were analysed using the developed SPE method, applying the optimised conditions.

The results in Fig. 6 indicate that the real sample recoveries on the LC-18 cartridge were the most acceptable as most analytes were in the $100 \pm 30 \%$ range. The samples seemed to exhibit matrix induced enhanced chromatographic effect on the LC-18 cartridge as all but one of the analytes produced recoveries greater than $100 \%$. Other cartridges produced recoveries of less than $100 \%$ for both the real sample and blank determinations. This indicates that there was either inefficient extraction or a matrix induced diminished chromatographic response. It is not unusual to obtain recoveries as high as $>200 \%$ in pesticide residue analysis in the presence of a real sample matrix as many labs worldwide have documented such cases [6].

\section{OPTIMUM MATRIX LOAD VOLUME VERSUS BREAKTHROUGH VOLUME}

The optimum matrix volume load, unlike the breakthrough volume was determined using an offline detection method and was determined for each specific analyte. In theory, as the sample is loaded onto the solid phase, it adsorbs the analytes and the organic matrix up to the point of saturation, where the solid phase reaches its retention capacity. This point of saturation is equivalent to the optimum matrix load volume. Any further analytes introduced to the solid phase beyond this point will not be quantitatively retained by the solid phase. The breakthrough volume, by definition is reached at the sample volume when amount of analytes entering and leaving the solid phase become equal, due to saturation of the solid phase by analytes introduced [25]. The results in Table $t$ above indicate that the $100 \mathrm{ml}$ real sample volume proved to be more robust as it produced the most precise recoveries within the $100 \pm 30 \%$ range for the organochlorine analytes compared to other sample volumes. The optimum matrix volume load curves for Lindane, pentachloronitrobenzene (PCNB) and BHC-alpha form the data extracted from Fig. 7 are shown below. 


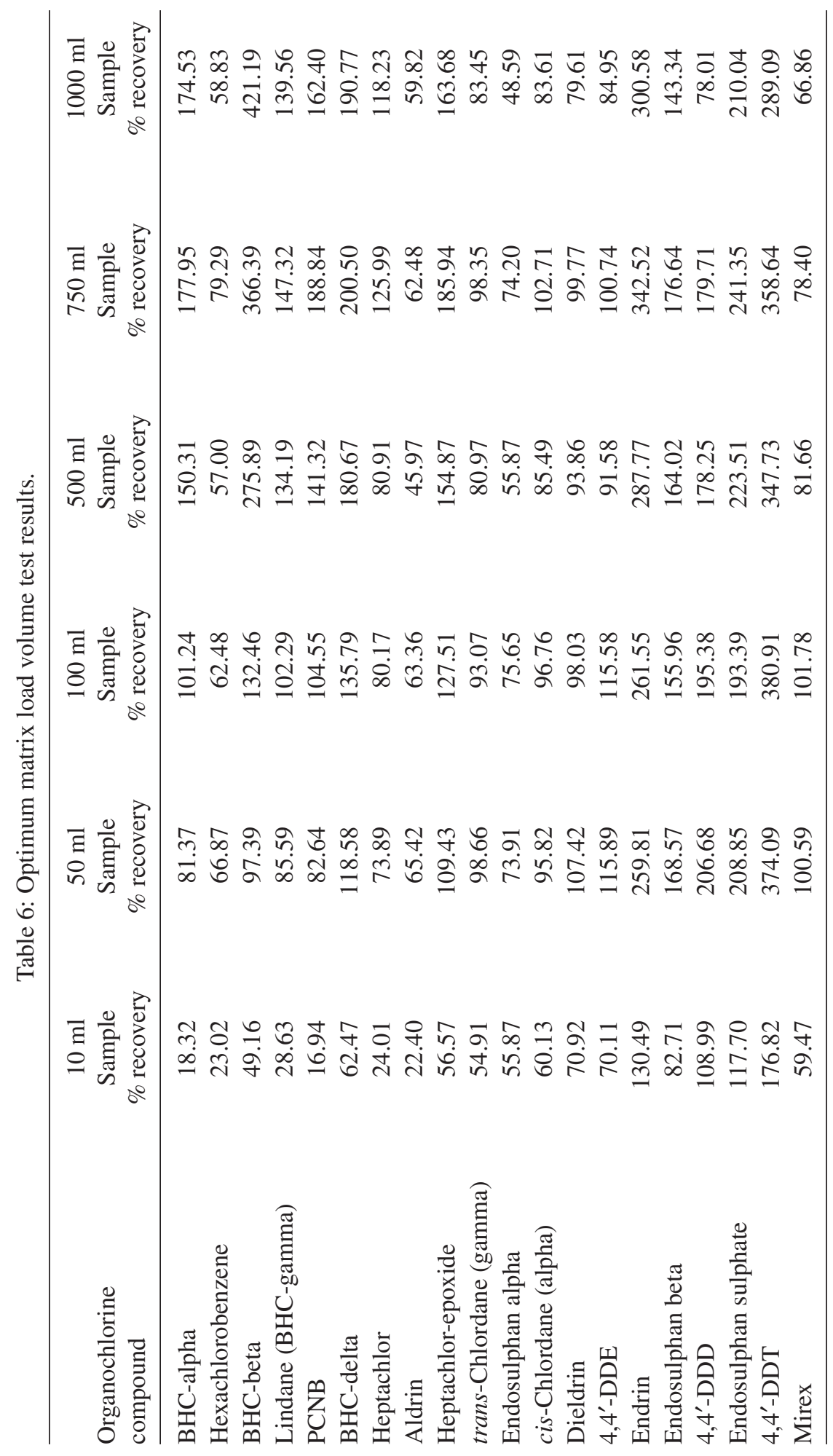




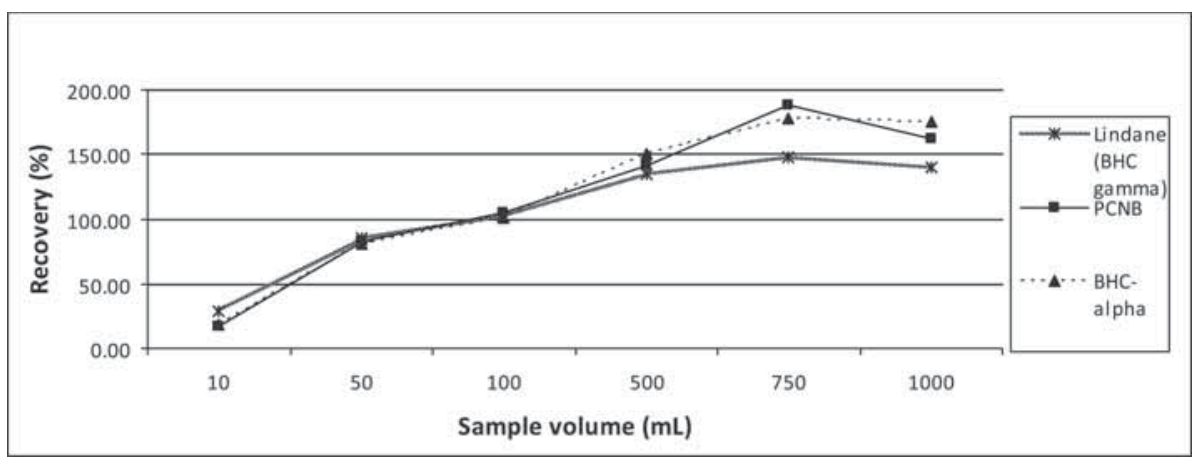

Figure 7: Optimum matrix load curve.

Both the breakthrough volume and optimum matrix volume load curve theoretically take the form of a sigmoid curve. The optimum matrix load volume for a particular analyte is the volume which produces a $100 \%$ recovery. One of the pitfalls of using optimum matrix volume load curve for multi-residue analysis is that the optimum volume varies for each particular analyte. For this research, $100 \mathrm{ml}$ was selected as the optimum matrix volume load curve and will be used for all further analyses.

\section{CHARACTERISATION OF THE MATRIX EFFECTS}

The chromatograms in Fig. 8 were injected successively into the GC. The adverse effects of the matrix on quantification and detection of analytes can to some extent be addressed by using SIM. In SIM, only data from the ion representing the ion signal of interest is generated, excluding information about the occurrence of other compounds. This, according to Kruve et al. [17], gives the illusion that other compounds that co-elute with the analyte of interest do not interfere with the results.

Often when the sample matrix is ionised together with the analytes, it presents problems associated with matrix signal suppression. Matrix signal suppression described by Choi et al. [31] can be clearly observed most notably on BHC and mirex, where the presence of the matrix lead to a significant reduction in the peak heights. This phenomenon is also called matrix induced response diminishment effects as the matrix induced a lower chromatographic response compared to the matrix-free extract. BHC-beta, on the other hand exhibited matrix induced response enhancement effects as the matrix caused an enhanced chromatographic response compared to the matrix-free extract [12].

Some of the clearly visible problems caused by the matrix in SIM analysis are

Bad separation

Loss of efficiency (sharp and narrow peaks)

Lower plate numbers

Loss of selectivity

Loss of resolution

Lower baseline separation

Broader peaks

Lower detection sensitivity

Higher background noise

Suppressed peak heights 


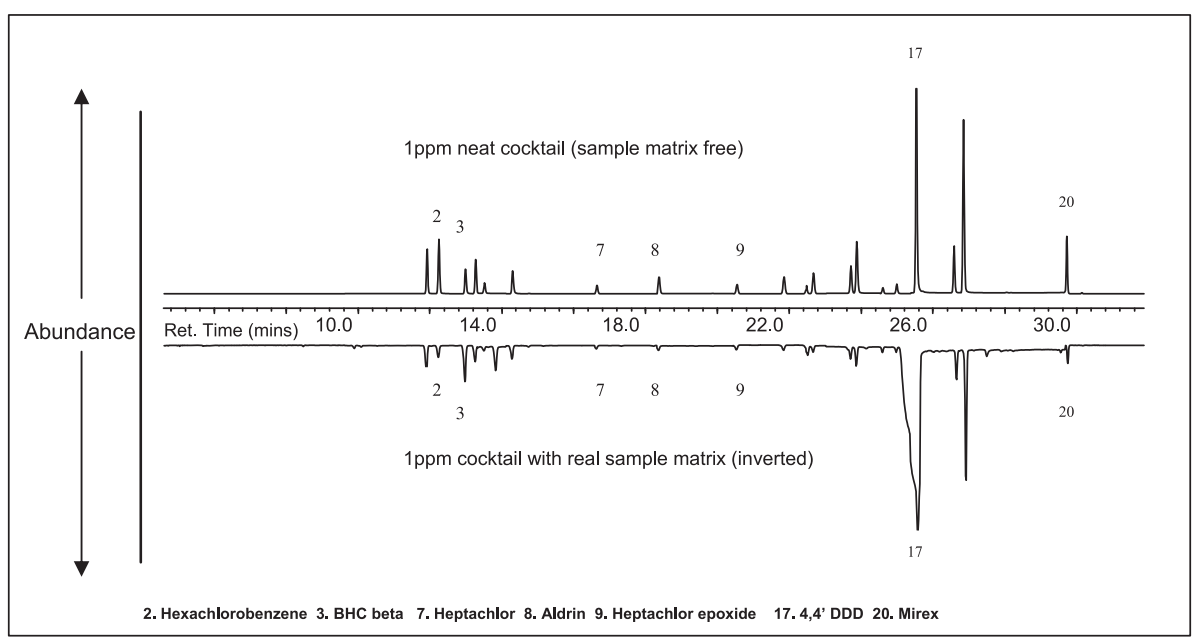

Figure 8: SIM chromatogram of spiked blank and real sample matrix.

Enhanced peak heights

Peak fronting and tailing

Other problems that result during data processing include

Integration errors

Reduced ruggedness (long term reproducibility)

Inaccurate quantification

Reporting false positive results

Reporting false negative results

Recording recoveries of up to $1000 \%$ as reported by Soboleva et al. [11].

\section{CONCLUSIONS}

SPE has proven to be one of the most effective techniques for the extraction of organochlorine pesticides from water samples. The automation of the SPE sample preparation techniques offers numerous and distinct advantages over conventional manual systems both in method development and routine sample analysis. The use of a highly selective SPE sorbent phase for the extraction of any particular analyte is essential for obtaining maximum analyte extraction and for attaining optimum recoveries. The sorbent mass and conditioning and elution volumes have a considerable effect on the efficiency of the SPE sample extraction procedure and hence should be carefully considered when developing a SPE analytical procedure. It is essential to determine the breakthrough volume or optimum matrix load volume for any particular cartridge as part of the validation, prior to analysis as the amount of matrix loaded onto the SPE cartridge has a resounding effect on the final recovery. The use of multi class residue analysis has proved to be an important technique with regards to cost and time saving, but is prone to matrix interference from within the synthetic matrix itself. The integrity of the validated SPE analytical procedure varies with the sample matrix composition. It is therefore recommended as far as practically possible, to validate methods using real samples with similar sample composition to the samples intended for subsequent progressive analysis. The matrix components affect the analysis at all the analytical steps including all the components 
of the entire chromatographic system hence adherence to optimum SPE cartridge treatment steps and regular maintenance of the GC system will go a long way to reduce matrix interference. It has been proven that the sample matrix clearly has a significant effect on the detection and quantification of target analytes and therefore should be investigated for each GC analytical procedure.

\section{REFERENCES}

[1] Hewavitharana, A.K., Matrix matching in liquid chromatography-mass spectrometry with stable isotope labelled internal standard- Is it necessary? Journal of Chromatography A, 1218, pp. 359-361, 2011. doi: http://dx.doi.org/10.1016/j.chroma.2010.11.047

[2] Kruve, A., Leito, I. \& Herodes, K., Combating matrix effects in LC/ESI/MS: the extrapolative dilution approach. Analytica Chimica Acta, 651, pp. 75-80, 2009. doi: http://dx.doi.org/10.1016/j.aca.2009.07.060

[3] Souverain, S., Rudaz, S. \& Veuthey, J-L., Matrix effect in LC-ESI-MS and LC-APCIMS with off-line and on-line extraction procedures. Journal of Chromatography A, 1058, pp. 61-64, 2004. doi: http://dx.doi.org/10.1016/j.chroma.2004.08.118

[4] Marin, J.M., Gracia-Lor, E., Sancho, J.V., Lopez, J.F. \& Hernandez, F., Application of ultra-high-pressure liquid chromatography-tandem mass spectrometry to the determination of multi-class pesticides in environmental and wastewater samples. Study of matrix effects. Journal of Chromatography A, 1216, pp. 1410-1412, 2009. doi: http:// dx.doi.org/10.1016/j.chroma.2008.12.094

[5] Hajslova, J. \& Zrostlikova, J., Matrix effects in (ultra)trace analysis of pesticide residues in food and biotic matrices. Journal of Chromatography A, 1000, pp. 181-189, 2003. doi: http://dx.doi.org/10.1016/S0021-9673(03)00539-9

[6] Hajslova, J., Holadova, K., Kocourek, V., Poustka, J., Godula, M., Cuhra, P. \& Kempny, M., Matrix induced effects: a critical point in the gas chromatographic analysis of pesticide residues. Journal of Chromatography A, 800, pp. 283-286, 1997. doi: http://dx.doi. org/10.1016/S0021-9673(97)01145-X

[7] Kelly, T., Gray, T.R. \& Huestis, M.A., Development and validation of a liquidchromatography-atmospheric pressure chemical ionisation-tandem mass spectrometry method for simultaneous analysis of 10 amphetamine-, metaphine- and 3,4-methylenedioxymethamphetamine-related (MDMA) analytes in human meconium. Journal of Chromatography B, 867, pp. 194-203, 2008. doi: http://dx.doi.org/10.1016/j. jchromb.2008.03.029

[8] Frenich, A.G., Martinez, J.L., Moreno, J.L.F. \& Romero-Gonzales, R., Compensation for matrix effects in gas chromatography-tandem mass spectrometry using a single point standard addition. Journal of Chromatography A, 1216, pp. 4798-4802, 2009. doi: http://dx.doi.org/10.1016/j.chroma.2009.04.018

[9] Huck, C.W. \& Bonn, G.K., Recent developments in polymer-based sorbents for solid-phase extraction. Journal of Chromatography A, 885, pp. 51-54, 2000. doi: http:// dx.doi.org/10.1016/S0021-9673(00)00333-2

[10] Sanchez-Brunete, C., Albero, B., Martin, G. \& Tadeo, J.L., Determination of pesticide residues by GC-MS using analyte protectants to counteract the matrix effect. Japan Society of Analytical Chemistry, 21, pp. 1291-1296, 2005.

[11] Soboleva, E., Ambrus, A. \& Jarju, O., Estimation of uncertainty of analytical results based on multiple peaks. Journal of Chromatography A, 1029, pp. 161-166, 2003. doi: http://dx.doi.org/10.1016/j.chroma.2003.10.139 
[12] Mastovska, K., Lehotay, S.J. \& Anastassiades, M., Combination of analyte protectants to overcome matrix effects in routine GC analysis of pesticide residues in food matrices. Analytical Chemistry, 77(24), pp. 8129-8134, 2005. doi: http://dx.doi.org/10.1021/ $\underline{\mathrm{ac} 0515576}$

[13] Benjitis, T., Dams, R., Lambert, W. \& De Leender, A., Countering matrix effects in environmental liquid chromatography-electrospray ionisation tandem mass spectrometry water analysis for endocrine disrupting chemicals. Journal of Chromatography A, 1029, pp. 153-155, 2004. doi: http://dx.doi.org/10.1016/j.chroma.2003.12.022

[14] Chiu, M.L., Lawi, W., Snyder, S.T., Wong, P.K., Liao, J.C. \& Gau, V., Matrix effects-a challenge toward automation of molecular analysis. Technology Review, 15(3), pp. 233-234, 2010.

[15] Chambers, E., Wagrowski-Diehl, D.M., Lu, Z. \& Mazzeo, R., Systematic and comprehensive strategy for reducing matrix effects in LC/MS/MS analyses. Journal of Chromatography B, 852, pp. 22-24, 2007. doi: http://dx.doi.org/10.1016/j.jchromb.2006.12.030

[16] Rogatsky, E. \& Stein, D., Evaluation of matrix effect and chromatography efficiency: new parameters for validation of method development. Journal of American Mass Spectrometry, 16, pp. 1757-1759, 2005. doi: http://dx.doi.org/10.1016/j.jasms.2005.07.012

[17] Kruve, A., Kunnapas, A., Herodes, K. \& Leito, I., Matrix effects in pesticide multiresidue analysis by liquid chromatography-mass spectrometry. Journal of Chromatography A, 1187, pp. 58-65, 2008. doi: http://dx.doi.org/10.1016/j.chroma.2008.01.077

[18] Kloepfer, A., Quintana, J.B. \& Thorsten, R., Operational options to reduce matrix effects in liquid chromatography-electrospray ionization-mass spectrometry analysis of aqueous environmental samples. Journal of Chromatography A, 1067, pp. 153-155, 2005. doi: http://dx.doi.org/10.1016/j.chroma.2004.11.101

[19] Marchi, I., Viette, V., Badoud, F., Fathi, M., Saugy, M., Rudaz, S. \& Veuthey, J., Characterisation and classification of matrix effects in biological samples analyses. Journal of Chromatography A, 1217, pp. 4071-4073, 2010. doi: http://dx.doi.org/10.1016/j. chroma.2009.08.061

[20] Bailey, R., Injectors for capillary gas chromatography and their application to environmental analysis. Journal of Environmental Monitoring, 7, pp. 1054-1057, 2005. doi: http://dx.doi.org/10.1039/b508334f

[21] Kicoyne, J. \& Fux, E., Strategies for the elimination of matrix effects in the liquid chromatography tandem mass spectrometry analysis of the lipophillic toxic okadaic acid and azaspiracid-1 in molluscan shellfish. Journal of Chromatography A, 1217, pp. 7123-7130, 2010. doi: http://dx.doi.org/10.1016/j.chroma.2010.09.020

[22] Poole, C.F., Matrix induced response enhancement in pesticide residue analysis by gas chromatography. Journal of Chromatography A, 1158, pp. 241-249, 2007. doi: http:// dx.doi.org/10.1016/j.chroma.2007.01.018

[23] Torres, M.E.H., Gonzalez, F.J.E., Cuadros-Rodreguez, L., Lopez, E.A. \& Vidal, J.L.M., Assessment of matrix effects in gas chromatography electron capture pesticide-residue analysis. Chromatographia, 57, pp. 657-659, 2003. doi: http://dx.doi.org/10.1007/ $\underline{\mathrm{BF} 02491745}$

[24] Rossi, D.T. \& Zhang, N., Automating solid-phase extraction: current aspects and future prospects. Journal of Chromatography A, 885, pp. 97-99, 2000. doi: http://dx.doi. org/10.1016/S0021-9673(99)00984-X

[25] Poole, C.F., Gunatilleka, A.D. \& Sethuraman, R., Contributions of theory to method development in solid-phase extraction. Journal of Chromatography A, 885, pp. 17-20, 2000. doi: http://dx.doi.org/10.1016/S0021-9673(00)00224-7 
[26] Nema, T., Chan, E.C.Y. \& Ho, P.C., Application of silica-based monolith as solid phase extraction cartridge for extracting polar compounds from urine. Talanta, 82, pp. 488-490, 2010. doi: http://dx.doi.org/10.1016/j.talanta.2010.04.063

[27] Gilson, Inc. Solid phase extraction solutions. USA, 2006. available at http://www.johnmorris.com.au/files/product/attachments/3429/239358_manual_instr.pdf

[28] Rimayi, C., Mtunzi, F., van Wyk, C. \& Odusanya, D., Method development for the influence of matrix on selected organochlorine pesticide residue analysis in surface water by GC-MS, Water Pollution XII. WIT Transactions on Ecology and Environment, ISSN: 1746-448X, 2012.

[29] Soboleva, E., Rathor, N., Mageto, A. \& Ambrus, A., Estimation of significance of matrix induced chromatographic effects. FAO/IAEA Training and reference centre for food and pesticide control. FAO/IAEA Agricultural and Biotechnology Laboratory. Principles and Practices of Method Validation, pp. 138-140, 2000. ISBN: 978-1-84755-175-7.

[30] Lehtonen, T., Peuravuori, J. \& Pihlaja, K., Characterisation of lake-aquatic humic matter isolated with two different sorbing solid techniques: tetramethylammonium hydroxide treatment and pyrolysis-gas chromatography/mass spectrometry. Analytica Chimica Acta, 424, pp. 91-103, 2000. doi: http://dx.doi.org/10.1016/S0003-2670(00)01141-7

[31] Choi, B.K., Hercules, D.M. \& Gusev, A.I., LC-MS/MS Signal suppression effects in the analysis of pesticides in complex environmental samples. Fresenius Journal of Analytical Chemistry, 369, pp. 370-373, 2001. doi: http://dx.doi.org/10.1007/s002160000661

[32] Miege, C., Bados, P., Brosse, C. \& Coquery, M., Method validation for the analysis of estrogens (including conjugated compounds) in aqueous matrices. Trends in Analytical Chemistry, 28(2), pp. 237-241, 2009. doi: http://dx.doi.org/10.1016/j.trac.2008.11.005

[33] Hayashi, Y., Matsuda, R., Haishima, Y., Yagami, T. \& Nakamura, A., Validation of HPLC and GC-MS systems for bisphenol-A leached from hemodialyzers on the basis of FUMI theory. Journal of Pharmaceutical and Biomedical Analysis, 28, pp. 421-423, 2002. doi: http://dx.doi.org/10.1016/S0731-7085(01)00698-7

[34] Quintana, J., Marti I. \& Ventura, F., Monitoring of pesticides in drinking and related waters in NE Spain with a multi residue SPE-GC-MS method including an estimation of uncertainty of the analytical results. Journal of Chromatography A, 938, pp. 1-8, 2001. doi: http://dx.doi.org/10.1016/S0021-9673(01)01168-2

[35] Stockl, D., D’Hondt, H. \& Thienpoint, L.M., Method validation across the disciplines- critical investigation of major validation criteria and associated experimental protocols. Journal of Chromatography B, 877, pp. 2180-2186, 2009. doi: http://dx.doi. org/10.1016/j.jchromb.2008.12.056

[36] Poole, C.F., New trends in solid-phase extraction. Trends in Analytical Chemistry, 22(6), pp. 362-373, 2003. doi: http://dx.doi.org/10.1016/S0165-9936(03)00605-8

[37] Zrostlikova, J., Hajslova, J., Poustka, J. \& Begany, P., Alternative calibration approaches to compensate the effect of co-extracted matrix components in liquid chromatographyelectrospray ionisation tandem mass spectrometry analysis of pesticide residues in plant materials. Journal of Chromatography A, 973, pp. 13-17, 2002. doi: http://dx.doi. org/10.1016/S0021-9673(02)01196-2

[38] Ferrer, I. \& Barcelo, D., Validation of new solid-phase extraction materials for the selective enrichment of organic contaminants from environmental samples. Trends in Analytical Chemistry, 18(3), pp. 181-182, 1999. doi: http://dx.doi.org/10.1016/S01659936(98)00108-3 